Published in final edited form as:

Cancer Epidemiol Biomarkers Prev. 2010 November ; 19(11): 2932-2941. doi:

10.1158/1055-9965.EPI-10-0686.

\title{
Associations of cumulative sun exposure and phenotypic characteristics with histologic solar elastosis
}

\author{
Nancy E. Thomas ${ }^{1,3}$, Anne Kricker ${ }^{4}$, Lynn From ${ }^{5}$, Klaus Busam ${ }^{6}$, Robert C. Millikan ${ }^{2,3}$, Mary \\ E. Ritchey ${ }^{2}$, Bruce K. Armstrong ${ }^{4}$, Julia Lee-Taylor ${ }^{7}$, Loraine D. Marrett ${ }^{8}$, Hoda Anton- \\ Culver $^{9}$, Roberto Zanetti ${ }^{10}$, Stefano Rosso ${ }^{10}$, Richard P. Gallagher ${ }^{11}$, Terence Dwyer ${ }^{12}$, \\ Chris Goumas ${ }^{4}$, Peter A. Kanetsky ${ }^{13}$, Colin B. Begg ${ }^{14}$, Irene Orlow ${ }^{14}$, Homer Wilcox ${ }^{15}$, \\ Susan Paine ${ }^{16}$, and Marianne Berwick ${ }^{16}$ The GEM Study Group \\ ${ }^{1}$ Department of Dermatology, University of North Carolina, Chapel Hill, North Carolina \\ ${ }^{2}$ Department of Epidemiology, University of North Carolina, Chapel Hill, North Carolina \\ ${ }^{3}$ Lineberger Comprehensive Cancer Center, University of North Carolina, Chapel Hill, North \\ Carolina \\ ${ }^{4}$ Sydney School of Public Health, The University of Sydney, Sydney, New South Wales, Australia \\ ${ }^{5}$ Women's College Hospital, Toronto, Ontario, Canada \\ ${ }^{6}$ Department of Pathology, Memorial Sloan-Kettering Cancer Center, New York, New York \\ ${ }^{7}$ Atmospheric Chemistry Division, National Center for Atmospheric Research, Boulder, Colorado \\ ${ }^{8}$ Cancercare Ontario, Toronto, Ontario, Canada \\ 'University of California, Irvine, California \\ ${ }^{10}$ Centro per la Prevenzione Oncologia Torino, Piemonte, Italy \\ ${ }^{11}$ British Columbia Cancer Agency, Vancouver, British Columbia, Canada \\ ${ }^{12}$ Murdoch Children's Research Institute, Royal Children's Hospital, Parkville, Victoria, Australia \\ ${ }^{13}$ University of Pennsylvania, Philadelphia, Pennsylvania \\ ${ }^{14}$ Department of Epidemiology and Biostatistics, Memorial Sloan-Kettering Cancer Center, New \\ York, New York \\ ${ }^{15}$ New Jersey Department of Health and Senior Services, Trenton, New Jersey \\ ${ }^{16}$ Department of Medicine, Division of Epidemiology, University of New Mexico, Albuquerque, New \\ Mexico
}

\section{Abstract}

Background-Solar elastosis adjacent to melanomas in histologic sections is regarded as an indicator of sun exposure although the associations of ultraviolet (UV) exposure and phenotype with solar elastosis are yet to be fully explored.

Methods-The study included 2,589 incident primary melanoma patients with assessment of histologic solar elastosis in the population-based Genes, Environment, and Melanoma study.

Copyright @ 2010 American Association for Cancer Research

Correspondence to: Nancy E. Thomas, MD PhD, Department of Dermatology, University of North Carolina, 3100 Thurston Bowles Bldg., CB\#7287, Chapel Hill, NC 27599. Phone: (919) 966-0785; Fax: (919) 966-6460; nthomas@ med.unc.edu. 
Ambient erythemal UV (UVE) at places of residence and sun exposure hours, including body sitespecific exposure, were collected. We examined the association of cumulative site-specific and non site-specific sun exposure hours and ambient UVE with solar elastosis in multivariable models adjusted for age, sex, center, pigmentary characteristics, nevi and, where relevant, body site.

Results-Solar elastosis was associated most strongly with site-specific UVE (OR for top exposure quartile, 5.20; 95\% CI, 3.40-7.96; $P$ for trend $<0.001$ ) and also with site-specific sun exposure (OR for top quartile, $5.12 ; 95 \% \mathrm{CI}, 3.35-7.83 ; P$ for trend $<0.001)$. Older age (OR at $>70$ years, $7.69 ; 95 \%$ CI, 5.14-11.52); $P$ trend < 0.001 ) and having more than 10 back nevi (OR, 0.77; 95\% CI, 0.61-0.97; $P=0.03)$ were independently associated with solar elastosis.

Conclusion-Solar elastosis had a strong association with higher site-specific UVE dose, older age and fewer nevi.

Impact-Solar elastosis could be a useful biomarker of lifetime site-specific UV. Future research is needed to explore whether age represents more than simple accumulation of sun exposure and the reason that people with more nevi may be less prone to solar elastosis.

\section{Keywords}

solar elastosis; UV; sun exposure; pigmentation; nevi

\section{Introduction}

Melanoma risk is positively associated with intermittent sun exposure and may be modestly inversely associated with a high continuous pattern of sun exposure (1). Solar elastosis, which is generally considered to be a biomarker for cumulative sun exposure, is frequently found adjacent to melanoma in histologic sections and is available for assessment by pathologists. The distinguishing feature of histologic solar elastosis is accumulation of abnormal elastotic fibers in the upper and middle dermis, which may be related to activation of the human elastin promoter by ultraviolet (UV) radiation (2) or to an influx of neutrophils that diffuse to the dermis in response to cytokine production after UVB exposure and degrade elastin (3). In albino hairless mice, the UV erythema spectrum was the most predictive for elastosis quantified by computerized image analysis (4). The importance of better understanding the relationship of age, sun exposure behavior and patient phenotype with solar elastosis among melanoma and other skin cancer patients is underscored by the use of solar elastosis as a biomarker in investigations of melanoma etiology (5-8) and outcome (9-13).

Recent epidemiological studies have explored environmental and personal determinants of skin damage, including whether there are separate effects of chronological aging and cumulative sun damage. These studies included people with BCC or SCC (14), melanoma (5) or were drawn from the general population (15) and examined either histologic solar elastosis $(5,14)$ or silicone cast score (5). While severe solar elastosis was found to occur more frequently on more heavily sun-exposed sites, no particular phenotypic determinant was identified in association with sun damage and only one study sought to identify the most predictive measure of sun exposure (14).

The GEM study was able to estimate cumulative UV dose at the site of the melanoma for all participants by integrating reported site-specific sun exposure behavior with estimated ambient UV irradiance at residential locations throughout life. In addition to the personal sun exposure histories, a range of pigmentary characteristics for patients and pathology information for the melanomas was available. In this paper, we aim to identify one or more measurements of sun exposure and the phenotypic or other personal characteristics that best predict histologic solar elastosis adjacent to the melanoma in incident primary cutaneous melanoma patients. 


\section{Materials and Methods}

The participants in this study were patients with incident first or subsequent primary cutaneous melanoma diagnosed from 1998 through 2003 in the Genes, Environment, and Melanoma (GEM) Study (16-18). GEM participants were ascertained from eight population-based cancer registries: two in Australia (New South Wales, Tasmania), two in Canada (British Columbia, Ontario), one in Italy (Torino), and three in the USA (Orange and San Diego Counties in California, New Jersey, and North Carolina).

The study protocol was approved by the Institutional Review Board (IRB) at the coordinating center, Memorial Sloan-Kettering Cancer Center, as well as the IRBs at each of the participating institutions. Physician approval was sought prior to contacting eligible participants, and all study participants provided informed consent. A total of 3,289 patients with incident primary cutaneous melanoma were enrolled from the eight centers. The participation rate was 53\%, and participants tended to be slightly younger and were more likely to be female compared with the entire ascertained population (17).

Each participant provided informed consent to obtain diagnostic slides of their melanoma for a standardized pathology review. Expert dermatopathologists scored adjacent solar elastosis on the same slides as the melanomas using a grading system (absent, mild/moderate, or severe) that correlated approximately to the chronic sun damage (CSD) levels proposed by Landi et al. (8). The kappa statistic using the three categories was 0.64 for scoring solar elastosis in a test set of 19 sections by three dermatopathologists who individually scored $70 \%, 20 \%$ and $10 \%$ of the total slide collection. For the analyses in this report, solar elastosis was grouped as 2 categories, present (mild, moderate, severe elastosis) versus absent.

Phenotypic variables were assessed by items in a self-administered questionnaire for participant's natural hair color as a teenager, eye color, and skin color on the inside of the upper arm (16), and instructions for a count of nevi (moles) on the back, which, for analysis, were categorized as 0-10 and more than 10. Questions in a telephone interview asked for propensity to burn on first exposure to sunlight in summer in 4 categories as 'no sunburn', 'mild burn then peel', 'painful burn then peel', 'severe burn with blistering' and ability to tan on repeated exposure to sunlight in 4 categories as 'go very brown and deeply tanned' to 'get no suntan or get freckled only'. Questions asked the number of each of painful and blistering sunburns at each decade of age in each of the warmer and cooler months and whether the melanoma site was usually burnt on these occasions; for analysis, these were dichotomized as any versus none.

\section{UV Measures}

Each participant was asked in the telephone interview for his or her sun exposure hours in the warmer and cooler months of each decade of life (ages 10, 20,30, and so on) up to his or her last completed decade of age. The warmer months in the Northern hemisphere were AprilSeptember and the cooler months October-March and the opposite for the Southern hemisphere. To estimate total exposure hours for a typical week, the hours were summed for outdoor hours reported between 9am and 5pm on work or school days and on non-working days across warmer and cooler months. Additional questions at each decade of age asked for the frequency of clothing coverage of the melanoma site and frequency of wearing sunscreen on the site when outdoors in the warmer and cooler months. The interview also sought a lifetime history from age 15 of hours spent in beach and water activities, which were known to be strongly associated with melanoma risk (16); exposure hours were summed for each age interval and the appropriate hours assigned to each decade of age. To estimate site-specific total sun exposure hours at each decade of age, we multiplied reported outdoor hours by the frequency of clothing coverage as follows: by 0 (always or almost always covered), 0.25 (not always but more than half the time), 0.50 (about half the time), 0.75 (less than half the time), 
or 1.00 (never or hardly ever). Total site-specific sun exposure hours were summed from age 5 and analyzed in categories defined by quartiles of subjects ranked in order by exposure. A measure of sunscreen use to the site was simply dichotomized as ever use or never use.

Using residential locations for each participant, ambient UV irradiance values were calculated for the warmer and cooler months at each decade of age. Erythemal UV (UVE) irradiances were calculated as wavelength integrated spectral irradiance between 250 and $400 \mathrm{~nm}$, weighted by the Commission Internationale de l'Eclairage erythemal sensitivity function (19), which gives greater weight to shorter wavelengths. The tropospheric UV-visible model 'TUV' (20) was used to calculate daily, location-specific irradiances as a function of solar zenith angle, ozone column, and surface elevation, as described by Lee-Taylor and Madronich (21). The model used a discrete ordinates method (22), and a pseudo-spherical correction (23). Corrections for variations in the Earth-Sun distance and for cloud cover (24) were applied. Ozone column and cloud reflectivity data were obtained from the satellite-borne Total Ozone Mapping Spectrometer (25-27) Version 7, for November 1978 to June 2000. Location specific UVE, UVA (320 - $400 \mathrm{~nm}$ unweighted) and UVB (280 - $320 \mathrm{~nm}$ unweighted) irradiance estimates from the 1978 to 1989 climatology were applied to all participant exposure dates before 1990, and values from the 1990 to 2000 climatology were applied to exposure dates from 1990 onward.

We estimated a cumulative site-specific UV dose for each of UVE, UVA and UVB as the total ambient UV for an assumed average 8 hours a day multiplied by the proportion of total sun exposure hours for which each participant reported exposure of the site. The seasonal values for warmer months and cooler months were combined for an annual dose and totaled over a lifetime from age 5. UVE, UVA and UVB were all highly correlated $\left(\mathrm{R}^{2}>0.99\right)$, although ambient UVB irradiance is more sensitive to the slant ozone column amount than is ambient UVA. Thus in this report, we present the analyses for UVE alone. The median and inter quartile range (IQR) for cumulative site-specific UVE in participants in each GEM center is presented in Figure 1. Site-specific beach and water UVE dose was calculated in a similar fashion to total dose, substituting beach and water hours for total outdoor hours.

\section{Statistical Analysis}

Age in these analyses was the patient's age at diagnosis of the melanoma for which the dermatopathologist scored solar elastosis in adjacent skin. GEM-wide quantiles were used in these analyses to categorize sun exposure variables into quarters of exposure for presentation in the Tables and into eighths for presentation in Figure 2, using cut points based on the exposure distribution in all participants. We examined the association of demographic, phenotypic and sun exposure variables with solar elastosis and estimated odds ratios (ORs) and 95\% confidence intervals (CI) for the presence of solar elastosis with reference to none, calculated in logistic regression models adjusted for age as a continuous variable, sex, and study center which was included as a covariate to adjust for differences in reviewing pathologists and percentages of diagnostic slides available in different centers. Tests for linear trend used the Wald test and modeled each covariate as a single continuous variable. All significance tests were two-sided. SAS (SAS Institute, Cary, NC, USA) version 9.2 was used for all analyses.

We assessed the strength of the association between solar elastosis and total reported hours of sun exposure, total site-specific hours (when the body site was exposed), ambient UVE at residential locations, total site-specific UVE (that is, integrating reported sun exposure behavior and UVE), each in quartiles with the lowest quartile as the reference, and site-specific beach and water UVE in exposure tertiles with no exposure as the reference. Site-specific hours and UVE were also modeled as continuous variables, and we present the odds per 1000 hours and per megajoule per meter ${ }^{2}\left(\mathrm{MJ} / \mathrm{m}^{2}\right)$ of exposure, respectively. The effect of age was 
examined in separate analyses as a categorical and a continuous variable. Before modeling with the continuous variables, we confirmed that there was a linear relationship with solar elastosis by plotting each variable in turn against the log odds of solar elastosis (25). The approach to assessing sun exposure effects was to model each sun exposure variable with adjustment for age, sex, center and to include as covariates the phenotypic characteristics back nevi, hair, eye and skin color, ability to tan and propensity to burn. Models for site-specific sun exposure also included a four-category variable for body site of the melanoma as head and neck, arms, legs, and trunk. To explore the extent to which age was an independent correlate of solar elastosis, we compared the estimates for age adjusted only for sex and center and when included in the multivariable model of sun exposure and phenotypic characteristics.

In addition to examining the size of any change in odds for solar elastosis and its statistical significance, we used the Akaike information criterion (AIC) to compare models and select the best fitting model to explain the observed data, given the candidate set of models (28). We calculated the corrected AIC $\left(\mathrm{AIC}_{\mathrm{c}}\right)$ values, which include a bias adjustment for the number of parameters relative to the sample size, and considered the best model as the one with the smallest $\mathrm{AIC}_{\mathrm{c}}$.

\section{Results}

Of the 3,289 participants with incident primary melanomas in the GEM study, 2,746 (83.5\%) had slides available for review. After pathology slide review, participants were excluded if solar elastosis was not scored due to insufficient adjacent tissue $(\mathrm{N}=95)$ or if the lesion was judged not to be melanoma by the reviewing pathologist $(\mathrm{N}=62)$. The $2,589(94.3 \%$ of 2,746$)$ melanomas included had histologic solar elastosis adjacent to their melanoma scored as absent, mild-moderate or severe; $1,805(69.7 \%)$ had solar elastosis present. The mean age of these 2,589 participants was 58.9 years and $56.4 \%$ were male. Most sites of melanoma were on the trunk (43.0\%), while the lower extremities (19.8\%), upper extremities $(18.5 \%)$ and head and neck $(18.1 \%)$ had approximately equal proportions; site was unknown for $0.6 \%$.

Risk of solar elastosis increased substantially with age to OR, 11.91 at 70+ years, with reference to 11-40 years (Table 1) and the OR for each year of age was 1.05 (95\% CI, 1.04-1.06). More men than women had solar elastosis (59.6\% vs. $49.0 \%)$ although the OR for solar elastosis in men, relative to women, was close to 1.0 when adjusted for age and study center. The odds for solar elastosis by tertile of age were reasonably similar for men and women together and separately although the increase with age was steeper in women than men (Table $1 ; P$ for interaction 0.05). Solar elastosis was more common on the head and neck and arms than the trunk or legs $(P<0.001)$ (Table 1$)$.

Dark hair (OR, 1.38; 95\% CI, 1.08-1.77) and light eye color (OR, 1.37; 95\% CI, 1.04-1.80) showed the strongest evidence of a positive association with solar elastosis when all phenotypic variables were fitted together in one model adjusted for age, sex and center. There was weaker evidence that odds of solar elastosis increased with red hair $(\mathrm{OR}=1.21)$ and skin that sunburned with blistering $(\mathrm{OR}=1.47)$ (Table 2). When site-specific UVE dose was also included in the multivariable model, the effects of hair and eye color weakened and there was evidence of a stronger trend in the odds for solar elastosis as tanning ability decreased or sunburning increased (Table 2). The effects of the pigmentary variables were substantially similar when examined in a multivariable model of all pigmentary variables (as in Table 2) and when each variable was examined separately adjusted only for age, sex and center (results not shown).

Having $>10$ nevi on the back, relative to $<10$, reduced the odds of solar elastosis $(\mathrm{OR}, 0.80$; 95\% CI 0.65-0.98; $P$ value $=0.03$ ) and was little changed when adjusted for pigmentary variables except the $P$ value was 0.07 (data not shown). The addition of site-specific UVE dose 
caused the effect of back nevi to strengthen a little to OR, 0.77 (95\% CI, 0.61-0.97) for >10 nevi $(P=0.03)$. In additional analyses, we found that the reduced odds for solar elastosis associated with the nevus propensity was mainly present for the trunk, arms and legs (OR, $0.75 ; 95 \%$ CI $0.59-0.96 ; P=0.02)$ and was not at all evident for the head and neck $(\mathrm{OR}, 1.12$; $95 \%$ CI $0.36-3.49 ; P=0.84)$, but the $P$ value for interaction was 0.61 .

Age was strongly and independently associated with elastosis in a model including site-specific UVE, phenotypic variables and back nevi. The addition of these variables, however, reduced the odds for age by $1 \%$ for each year of age from OR, 1.06 to OR, 1.05 (95\% CI 1.04-1.06) and by $35 \%$ at 70 years and older from OR, 11.91 to OR, 7.69 (95\% CI 5.14-11.52), relative to $11-40$ years, for men and women together and somewhat more in women $(-40 \%)$ than men $(-25 \%)$ of this age.

Solar elastosis was associated most strongly with estimates of cumulative sun exposure to the body site. Site-specific sun exposure hours increased the odds for solar elastosis to an OR of 5.12 for the top exposure quartile (Q4; $P$ value for trend $<0.001$; Table 3$)$ and the OR for the continuous measure was $1.05(95 \% \mathrm{CI}, 1.03-1.06 ; P<0.001)$ for each 1000 site-specific hours. Inclusion of ambient UVE in this measure to give site-specific UVE dose increased the OR to 5.20 for Q4 (Table 3) and the OR for the continuous measure was 1.11 (95\% CI, 1.08-1.14; $P<0.001)$ per $\mathrm{MJ} / \mathrm{m}^{2}$. Total outdoor hours without consideration of exposure of the body site or UVE increased the odds for solar elastosis to an OR of 2.13 for Q4 $(P$ value for trend < 0.001 ) while cumulative ambient UVE, as a non-specific measure based only on places of residence, had no evident association with solar elastosis $(\mathrm{OR}, 1.29$ for Q4; $P$ for trend $=0.49)$ although the inclusion of center as a covariate to account for different pathology reviewers in different centers would have substantially limited between subject variation in UVE in this analysis. We also examined site-specific UV dose for hours spent in beach and water activities as a measure of recreational sun exposure. The odds for the highest exposure tertile, with reference to no beach and water activities, was OR, 2.37; 95\% CI 1.70-3.30; P for trend $<0.001$. All sun exposure models were adjusted for age, sex, center and all phenotypic variables; sitespecific measures were also adjusted for body site.

Site-specific UVE appears to be the best predictor of solar elastosis in these data. For exposure in quartiles (Table 3), the $\mathrm{AIC}_{\mathrm{c}}$ was lowest for site-specific $\mathrm{UVE}\left(\mathrm{AIC}_{\mathrm{c}}=2006\right)$, although little different to the value for site-specific sun exposure hours $\left(\mathrm{AIC}_{\mathrm{c}}=2007\right)$. Total sun exposure hours without site specificity did not fit the data well $\left(\mathrm{AIC}_{\mathrm{c}}=2279\right)$. The evidence that sitespecific UVE was the best measure of UV exposure was stronger when exposure measures were modeled as continuous variables $\left(\mathrm{AIC}_{\mathrm{c}}=1994\right.$ for site-specific UVE, 1999 for sitespecific exposure hours and 2278 for total exposure hours only). This hierarchy of cumulative sun exposure measures is clearly evident when ORs for exposure in 8 categories are plotted graphically for all body sites together (Figure 2).

In separate analyses by body site, the odds for solar elastosis were high for site-specific UVE to the head and neck (OR, 1.70; 95\% CI, 0.26-11.13 for Q2; OR, 4.13; 95\% CI, 0.71-24.14 for Q3; OR, 17.95; 95\% CI, 2.52-128.15 for Q4; $P=0.002$ ) while the odds for the trunk, arms and legs were overall similar to the estimates in all participants (OR, 1.70; 95\% CI, 1.26-2.30 for Q2; OR, 2.24; 95\% CI, 1.60-3.12 for Q3; OR, 4.88; 95\% CI, 3.13-7.62 for Q4; $P<0.001$ ). The $P$ for interaction by body site was 0.23 . Almost all lesions on the head and neck $(94 \%)$ were positive for solar elastosis and most (59\%) were rated as severe. After excluding head and neck lesions, the estimates for exposure categorized in eighths were very similar to those presented for all lesions in Figure 2. All models were adjusted for age, sex, center and phenotypic characteristics. 
Sunburn history showed no evidence of an association with solar elastosis in separate analyses of any site-specific painful sunburn (OR, 1.02; 95\% CI, 0.79-1.32; $P=0.88)$ or blistering sunburn (OR, $0.95 ; 95 \% \mathrm{CI}, 0.70-1.31 ; P=0.77)$ relative to none in each case. Site-specific sunscreen use similarly had no apparent association with solar elastosis (OR for ever use, 1.18; 95\% CI, 0.94-1.48; $P=0.15$ ). In separate analyses of site-specific UVE in users and non-users of sunscreen on the site, ORs in users were lower (OR, 1.32; 95\% CI, 0.89-1.98 for Q2; 2.22; 95\% CI, 1.40-3.53 for Q3; 4.53; 95\% CI, 2.40-8.53 for Q4; $P$ for trend <0.001) than in nonusers for each exposure category, especially Q4 (OR, 2.06; 95\% CI, 1.21-3.50 for Q2; 2.81; 95\% CI, 1.64-4.83 for Q3; 8.47; 95\% CI, 4.16-17.23 for Q4; $P$ for trend $<0.001$ ) ( $P$ for interaction $=0.09$ ). All analyses were adjusted for age, sex, center and phenotypic variables; the model for sunburn also included site-specific UVE and body site, and the model examining site-specific UVE by sunscreen use also included body site.

\section{Discussion}

We found that cumulative site-specific UVE dose had a strong positive dose-response relationship with histologic solar elastosis adjacent to melanomas. Additionally, site-specific UVE dose was the best of a number of sun exposure measurements in accounting for solar elastosis, judging by the AIC statistic. We also found that solar elastosis had a strong positive association with age even after adjusting for cumulative site-specific UVE dose and that elastosis occurred more frequently in skin on the sun-exposed head and neck and the arms than on the lesser exposed trunk and legs. Having more than 10 nevi on the back reduced the overall chance of solar elastosis. In addition to high cumulative sun exposure, our results indicate that older age and a tendency to fewer nevi were the personal characteristics that increased the chance of solar elastosis.

The strong positive association of histologic solar elastosis with cumulative sun exposure in GEM supports similar findings in previous studies that examined histologic solar elastosis in skin cancer patients $(5,14)$ or photoaging ratings in silicone skin casts in the general population (15). The link to cumulative exposure was consistent across sunlight environments from high levels of Queensland sun (5) to low levels in New Hampshire (14), across a wide latitude range at $27^{0} \mathrm{~S}$ to $43^{0} \mathrm{~S}(15)$, and now internationally in our study. All studies measured sun exposure behavior individually in participants, but only GEM and the New Hampshire study (14) used site-specific measurements; others used a composite of weekend outdoor hours and residential UVE (15) or broad categories of cumulative exposure (5). Rates of sun damage were high, with moderate or severe elastosis in 70\% of Queensland (5) and GEM melanoma patients and $90 \%$ or more of New Hampshire BCC and SCC patients (14). Australian residents too had high skin damage scores (3.9 and 4.9 for each hand on a scale of 6) (15). The finding in GEM that high levels of sun exposure increased the chance of solar elastosis more on the head and neck than at other body sites was in agreement with studies that directly measured sun exposure $(5,14)$ or examined tissue $(5,12,29)$ at different body sites. Unlike the very strong and consistent association with cumulative sun exposure, site-specific lifetime recreational sun exposure increased the odds for solar elastosis but not strongly in our study and only for intermediate exposure levels in the New Hampshire study (14) or not significantly in Queensland patients (5).

Karagas et al. (14) were the first to report the strong association of solar elastosis with cumulative sun exposure to the site, but did not present results for non-specific measures and did not incorporate ambient UV, as we did. The strong associations we observed in GEM for site-specific exposure can be ascribed to reducing exposure misclassification and giving weight to the environment by incorporating ambient UV and site-specific exposure hours into a single measure. We have shown that adding environmental UVE to self-reported sun exposure hours can improve site-specific sun exposure measurement. The results of our study support the use 
of solar elastosis as a biomarker of cumulative sun exposure in epidemiological studies that collect skin tissue.

The positive correlation of age with solar elastosis agrees with the greater prevalence at older ages in New Hampshire, especially in women (14), and in Australia $(5,15,30)$. Both age and cumulative sun exposure were independently associated with solar elastosis in our study and potentially also in the US and Australian studies, which both mention that adjusting for age did not weaken the relationship with cumulative sun exposure. They do not, however, report estimates for age when adjusted for sun exposure $(14,15)$. The strong association with age may be due to a contribution of intrinsic aging to solar elastosis or to the accumulation of UV exposure with age that is not fully addressed by adjusting for sun exposure in the epidemiological studies, or to other unmeasured variables. We expect that future studies, perhaps incorporating site-specific UVE measurements, will attempt to disentangle the contributions of aging and sun exposure in causing sun-related conditions such as solar elastosis or skin cancer.

Our observation that a high nevus count on the back in the GEM study reduced the odds of solar elastosis by $20 \%$ is consistent with the Queensland study in which there was some evidence that greater numbers of nevi may have reduced the odds for solar elastosis, based on patient numbers (5), and the Australian general population study in which having any nevi reduced the odds of sun damage to 0.39 (95\% CI, 0.23-0.66) (15).

Sun exposure is known to be positively associated with both nevus count (31) and presence of solar elastosis. Thus we could reasonably expect the nevus propensity to be associated with a greater likelihood of solar elastosis. Instead, the available evidence indicates that solar elastosis was less likely in the presence of a higher nevus count (5) even after controlling for sun exposure and sun sensitivity (see Table 3 and (15)). Since nevi are genetically determined (32-34), there may be a genetic component to the resistance to solar elastosis. In our study, the reduced odds ratio for solar elastosis associated with the nevus propensity was due mainly to solar elastosis on the trunk, arms and legs. We suggest that being less prone to solar elastosis and possibly other forms of sun damage such as solar keratoses (14) may be another element in the dual pathway hypothesis under which a person predisposed to nevi requires less sun exposure to develop melanoma $(35,36)$. The hypothesis does not currently specify an element of resistance to sun damage in this pathway.

Our results for phenotype are moderately supportive of the importance of a sun sensitive phenotype for solar elastosis (15). Reduced tanning ability and a severe sunburn response to unprotected exposure were each associated with solar elastosis in our study and strengthened on adjustment for sun exposure, although not significantly so, but there was no strong and consistent effect of skin or hair color. Similarly, tanning and skin color (assessed by colorimeter) had no evident effect either way on solar elastosis in BCC and SCC patients (14) while the Queensland study did not report on pigmentary characteristics (5). There may not yet be sufficient evidence for the role of host phenotype in the development of skin damage. The lack of prominence for phenotype, especially fair skin, in association with solar elastosis in studies of normal skin in skin cancer patients may be a consequence of most patients having fair skin (88\% had fair or very fair skin in GEM).

Sunburn is considered to be a measure of intermittent sun exposure. Unlike the strong relationship with cumulative site-specific UVE, sunburn had no evident association with solar elastosis in our study or the New Hampshire study (14). A possible explanation is that people who burn readily may accumulate less time in the sun and thus are less likely to experience marked solar elastosis. Having sunburns or blistering sunburns, however, had a two-fold or more increased odds of sun damage after adjusting for cumulative whole body sun exposure, 
but not pigmentary characteristics, in the Australian general population study (15). There is no ready explanation for this inconsistency. Possibilities include differences in the nature of skin damage when measured by skin cast or histologic assessment, differences due to the body site examined since the back of the hand is known to be less susceptible to skin cancer, or the possibility of confounding with an underlying genetic component (37) that is more common in people with skin cancer than in the general population. Another possibility is that the high UV environment of the Australian population offered a greater opportunity to experience sunburn (87\% reported any sunburn, 66\% blistering sunburn) (15) than in GEM (64\% any sunburn, 45\% blistering sunburn) or New Hampshire (57\% painful sunburn) (14). We interpret the lack of an effect of sunburn on solar elastosis in the skin cancer studies as further support for the contribution of cumulative sun exposure, and not intermittent sun exposure, to solar elastosis.

Our finding that the risk of solar elastosis was higher for site-specific UVE in people who reported no sunscreen use on the body site than in users is the first report of an apparently protective effect of sunscreens against this type of sun damage. It is, however, consistent with a strong inverse dose-response relationship between amount of sunscreen used regularly and development of new solar keratoses and remission of existing ones (38), and with prevention of SCC, but not BCC, by recent use of sunscreens (39).

Among the advantages of this report are the standardized histopathology review by expert pathologists of all melanoma slides including the scoring of solar elastosis, the study's large international population base, accounting for potential confounding by phenotype, and the detailed sun exposure measurements integrating behavior with objective ambient UV measurements and restricting exposure to times when the site was exposed to the sun. All participants had melanoma and were unlikely to be aware of whether they had solar elastosis or not, thus minimizing the potential for recall bias.

The measures incorporating behavior, however, may have inaccuracies due to error in selfreported exposure hours over a lifetime. Our site-specific UVE dose may have other sources of error because it included only exposure hours between 9am and 5pm (up to 15\% of ambient UVE falls outside of 9am- 5pm, see Madronich, 1993 (40)) and could make no allowance for variation in ambient UVE irradiance between 9am and $5 \mathrm{pm}$.

Variables used in the modeling were not predictive of missing data for solar elastosis, except for center location, which was mainly due to the different percentages of participants with diagnostic slides available for review. Although all study participants had melanoma and thus our results might be considered not to apply more widely, the consistency of our results with the strong association of cumulative UVR dose and skin damage reported in the general population (15) supports their wider applicability.

We found that solar elastosis was strongly associated with cumulative lifetime site-specific UV dose. This relationship is biologically plausible. Our study was the first to demonstrate the extent of the improvement gained in evaluating the relationship between sun exposure and solar elastosis by using site-specific measurements and, additionally, the stronger effect estimates that resulted from accounting for ambient UV. We conclude that solar elastosis could be a useful biomarker of cumulative sun exposure in epidemiological studies that collect skin biopsies. The strong relationship of solar elastosis with age could indicate the possibility of an explanation other than a simple accumulation of sun exposure over time. Finally, the inverse, independent relationship with number of nevi may suggest that people with a genetic predisposition to nevi may be less prone to sun damage. Future studies would usefully include possible genetic markers of relevance, including any that may be related to solar elastosis. 


\section{Acknowledgments}

The study was conducted by the GEM Study Group: Coordinating Center, Memorial Sloan - Kettering Cancer Center, New York, NY: Marianne Berwick (PI, currently at the University of New Mexico), Colin B. Begg (Co-PI), Irene Orlow (Co-Investigator), Urvi Mujumdar (Project Coordinator), Amanda J. Hummer (Biostatistician), Nandita Mitra (Biostatistician), Klaus Busam (Dermatopathologist), Pampa Roy (Laboratory Technician), Rebecca Canchola (Laboratory Technician), Brian Clas (Laboratory Technician), Javier Cotignola (Laboratory Technician), and Yvette Monroe (Interviewer). Study centers included the following: The University of Sydney and The Cancer Council New South Wales, Sydney, Australia: Bruce K. Armstrong (PI), Anne Kricker (Co-PI), Melisa Litchfield (Study Coordinator); Menzies Centre for Population Health Research, University of Tasmania, Hobart, Australia: Terence Dwyer (PI, currently at the Murdoch Research Institute, Royal Children's Hospital, Parkville, Victoria), Paul Tucker (Dermatopathologist), Nicola Stephens (Study Coordinator); British Columbia Cancer Agency, Vancouver, Canada: Richard P. Gallagher (PI), Teresa Switzer (Coordinator); Cancer Care Ontario, Toronto, Canada: Loraine D. Marrett (PI), Elizabeth Theis (Co-Investigator), Lynn From (Dermatopathologist), Noori Chowdhury (Coordinator), Louise Vanasse (Coordinator), Mark Purdue (Research Officer), David Northrup (Manager for CATI); Center for the Prevention of Cancer - Turin, Piedmont, Italy: Roberto Zanetti (PI), Stefano Rosso (Data Manager), Carlotta Sacerdote (Coordinator); University of California, Irvine: Hoda Anton-Culver (PI), Nancy Leighton (Coordinator), Maureen Gildea (Data Manager); University of Michigan, Ann Arbor: Stephen B. Gruber (PI), Joe Bonner (Data Manager), Joanne Jeter (Coordinator); New Jersey Department of Health and Senior Services, Trenton: Judith Klotz (PI), Homer Wilcox (Co-PI), Helen Weiss (Coordinator); University of North Carolina, Chapel Hill: Robert C. Millikan (PI), Nancy Thomas (Co- Investigator), Dianne Mattingly (Coordinator), Jon Player (Laboratory Technician), Chiu-Kit Tse (Data Analyst); University of Pennsylvania: Timothy R. Rebbeck (PI), Peter Kanetsky (Co-Investigator), Amy Walker (Laboratory Technician), Saarene Panossian (Laboratory Technician); Consultants: Harvey Mohrenweiser, University of California, Irvine; Richard Setlow, Brookhaven National Laboratory, Upton, NY. UV data consultants: Dr Julia Lee Taylor and Dr Sasha Madronich, National Centre for Atmospheric Research, Boulder, Co.

Financial support: National Cancer Institute (NCI) grants R01 CA112243, R01 112524, R01 CA112243-05S1, R01 CA112524-05S2, U01 CA83180, and ES014635; University of Sydney Medical Foundation Program grant (Bruce Armstrong); NCI grant CA098438 (Colin Begg); Michael Smith Foundation for Health Research Infrastructure Award (Richard Gallagher); Lineberger Comprehensive Cancer Center Core grant P30 CA16086 (Robert Millikan).

\section{References}

1. Gandini S, Sera F, Cattaruzza MS, et al. Meta-analysis of risk factors for cutaneous melanoma: II. Sun exposure. Eur J Cancer 2005;41:45-60. [PubMed: 15617990]

2. Uitto J. The role of elastin and collagen in cutaneous aging: intrinsic aging versus photoexposure. $\mathrm{J}$ Drugs Dermatol 2008;7:s12-6. [PubMed: 18404866]

3. Rijken F, Bruijnzeel PL. The pathogenesis of photoaging: the role of neutrophils and neutrophil-derived enzymes. J Investig Dermatol Symp Proc 2009;14:67-72.

4. Kligman LH, Sayre RM. An action spectrum for ultraviolet induced elastosis in hairless mice: quantification of elastosis by image analysis. Photochem Photobiol 1991;53:237-42. [PubMed: 2011628]

5. Lee EY, Williamson R, Watt P, et al. Sun exposure and host phenotype as predictors of cutaneous melanoma associated with neval remnants or dermal elastosis. Int J Cancer 2006;119:636-42. [PubMed: 16572428]

6. Maldonado JL, Fridlyand J, Patel H, et al. Determinants of BRAF mutations in primary melanomas. J Natl Cancer Inst 2003;95:1878-90. [PubMed: 14679157]

7. Curtin JA, Fridlyand J, Kageshita T, et al. Distinct sets of genetic alterations in melanoma. N Engl J Med 2005;353:2135-47. [PubMed: 16291983]

8. Landi MT, Bauer J, Pfeiffer RM, et al. MC1R germline variants confer risk for BRAF-mutant melanoma. Science 2006;313:521-2. [PubMed: 16809487]

9. Barnhill RL, Fine JA, Roush GC, Berwick M. Predicting five-year outcome for patients with cutaneous melanoma in a population-based study. Cancer 1996;78:427-32. [PubMed: 8697387]

10. Barnhill RL, Katzen J, Spatz A, Fine J, Berwick M. The importance of mitotic rate as a prognostic factor for localized cutaneous melanoma. J Cutan Pathol 2005;32:268-73. [PubMed: 15769275]

11. Berwick M, Armstrong BK, Ben-Porat L, et al. Sun exposure and mortality from melanoma. J Natl Cancer Inst 2005;97:195-9. [PubMed: 15687362]

12. Vollmer RT. Solar elastosis in cutaneous melanoma. Am J Clin Pathol 2007;128:260-4. [PubMed: 17638660] 
13. Heenan PJ, English DR, Holman CD, Armstrong BK. Survival among patients with clinical stage I cutaneous malignant melanoma diagnosed in Western Australia in 1975/1976 and 1980/1981. Cancer 1991;68:2079-87. [PubMed: 1913557]

14. Karagas MR, Zens MS, Nelson HH, et al. Measures of cumulative exposure from a standardized sun exposure history questionnaire: a comparison with histologic assessment of solar skin damage. Am J Epidemiol 2007;165:719-26. [PubMed: 17204514]

15. Lucas RM, Ponsonby AL, Dear K, et al. Associations between silicone skin cast score, cumulative sun exposure, and other factors in the ausimmune study: a multicenter Australian study. Cancer Epidemiol Biomarkers Prev 2009;18:2887-94. [PubMed: 19843682]

16. Kricker A, Armstrong BK, Goumas C, et al. Ambient UV, personal sun exposure and risk of multiple primary melanomas. Cancer Causes Control 2007;18:295-304. [PubMed: 17206532]

17. Millikan RC, Hummer A, Begg C, et al. Polymorphisms in nucleotide excision repair genes and risk of multiple primary melanoma: the Genes Environment and Melanoma Study. Carcinogenesis 2006;27:610-8. [PubMed: 16258177]

18. Begg CB, Hummer AJ, Mujumdar U, et al. A design for cancer case-control studies using only incident cases: experience with the GEM study of melanoma. Int J Epidemiol 2006;35:756-64. [PubMed: 16556646]

19. McKinlay, AF.; Diffey, BL. A reference action spectrum for ultraviolet induced erythema in human skin. In: Passchier, WR.; Bosnjakovic, BFM., editors. Human exposure to ultraviolet radiation: risks and regulations. Amsterdam: Elsevier; 1987.

20. Madronich, S.; Flocke, S. Theoretical estimation of biologically effective UV radiation at the Earth's surface, in Solar Ultraviolet Radiation - Modeling, Measurements and Effects. In: Zerefos, C., editor. NATO ASI Series. Berlin: Springer-Verlag; 1997.

21. Lee-Taylor, J.; Madronich, S.; Mayer, B.; Fischer, C. A Climatology of UV Radiation, 1979 - 2000, 65S -65N. In: Gao, W.; Schmoldt, D.; Slusser, J., editors. Chapter 1, UV Radiation in Global Climate Change: Measurements, Modeling and Effects on Ecosystems. Springer-Verlag and Tsinghua University Press; 2010.

22. Stamnes K, Tsay S, Wiscombe W, Jayaweera K. A numerically stable algorithm for discrete-ordinatemethod radiative transfer in multiple scattering and emitting layered media. Appl Opt 1988;27:25029. [PubMed: 20531783]

23. Petropavlovskikh, I. A numerically stable algorithm for discrete-ordinate-method radiative transfer in multiple scattering and emitting layered media [PhD]. Boulder: U. of Brussels and NCAR/CT-159; 1995.

24. Eck TF, Bhartia PK, Kerr JB. Satellite estimation of spectral UVB irradiance using TOMS derived total ozone and UV reflectivity. Geophys Res Lett 1995;22:611-4.

25. McPeters, RD.; Bhartia, K.; Krueger, AJ., et al. NASA Tech Pub 1998-206895. Greenbelt, MD: Goddard Space Flight Center; 1998. Earth Probe Total Ozone Mapping Spectrometer (TOMS) Data Products User's Guide.

26. McPeters, RD.; Bhartia, K.; K, AJ., et al. NASA Reference Publication 1384. National Aeronautics and Space Administration; Washington, D.C: 1996. Total Ozone Mapping Spectrometer (TOMS) Data Products User's Guide.

27. Herman JR, Krotkov N, Calarier E, Larko D, Labow G. Distribution of UV radiation at the Earth's surface from TOMS-measured UV-backscattered radiances. J Geophys Res 1999;104:12059-76.

28. Burnham, KP.; Anderson, DR. Model selection and inference: a practical information-theoretic approach. second edition. New York: Springer-Verlag; 2010.

29. Black WC. Residual dysplastic and other nevi in superficial spreading melanoma. Clinical correlations and association with sun damage. Cancer 1988;62:163-73. [PubMed: 3383113]

30. Battistutta D, Pandeya N, Strutton GM, et al. Skin surface topography grading is a valid measure of skin photoaging. Photodermatol Photoimmunol Photomed 2006;22:39-45. [PubMed: 16436180]

31. Gallagher RP, Rivers JK, Lee TK, et al. Broad-spectrum sunscreen use and the development of new nevi in white children: A randomized controlled trial. JAMA 2000;283:2955-60. [PubMed: 10865273]

32. Bataille V, Snieder H, MacGregor AJ, Sasieni P, Spector TD. Genetics of risk factors for melanoma: an adult twin study of nevi and freckles. J Natl Cancer Inst 2000;92:457-63. [PubMed: 10716963] 
33. Falchi M, Spector TD, Perks U, Kato BS, Bataille V. Genome-wide search for nevus density shows linkage to two melanoma loci on chromosome 9 and identifies a new QTL on $5 q 31$ in an adult twin cohort. Hum Mol Genet 2006;15:2975-9. [PubMed: 16928783]

34. Zhu G, Duffy DL, Eldridge A, et al. A major quantitative-trait locus for mole density is linked to the familial melanoma gene CDKN2A: a maximum-likelihood combined linkage and association analysis in twins and their sibs. Am J Hum Genet 1999;65:483-92. [PubMed: 10417291]

35. Whiteman DC, Watt P, Purdie DM, et al. Melanocytic nevi, solar keratoses, and divergent pathways to cutaneous melanoma. J Natl Cancer Inst 2003;95:806-12. [PubMed: 12783935]

36. Whiteman DC, Parsons PG, Green AC. p53 expression and risk factors for cutaneous melanoma: a case-control study. Int J Cancer 1998;77:843-8. [PubMed: 9714052]

37. Nan H, Kraft P, Qureshi AA, et al. Genome-wide association study of tanning phenotype in a population of European ancestry. J Invest Dermatol 2009;129:2250-7. [PubMed: 19340012]

38. Thompson SC, Jolley D, Marks R. Reduction of solar keratoses by regular sunscreen use. N Engl J Med 1993;329:1147-51. [PubMed: 8377777]

39. Green A, Williams G, Neale R, et al. Daily sunscreen application and betacarotene supplementation in prevention of basal-cell and squamous-cell carcinomas of the skin: a randomised controlled trial. Lancet 1999;354:723-9. [PubMed: 10475183]

40. Madronich, S. UV radiation in the natural and perturbed atmosphere. In: Tevini, M., editor. Chapter 2, UV-B radiation and ozone depletion: effects on humans, animals, plants, microorganisms, and materials. Boca Raton: Lewis Publishers; 1993. p. 17-68. 


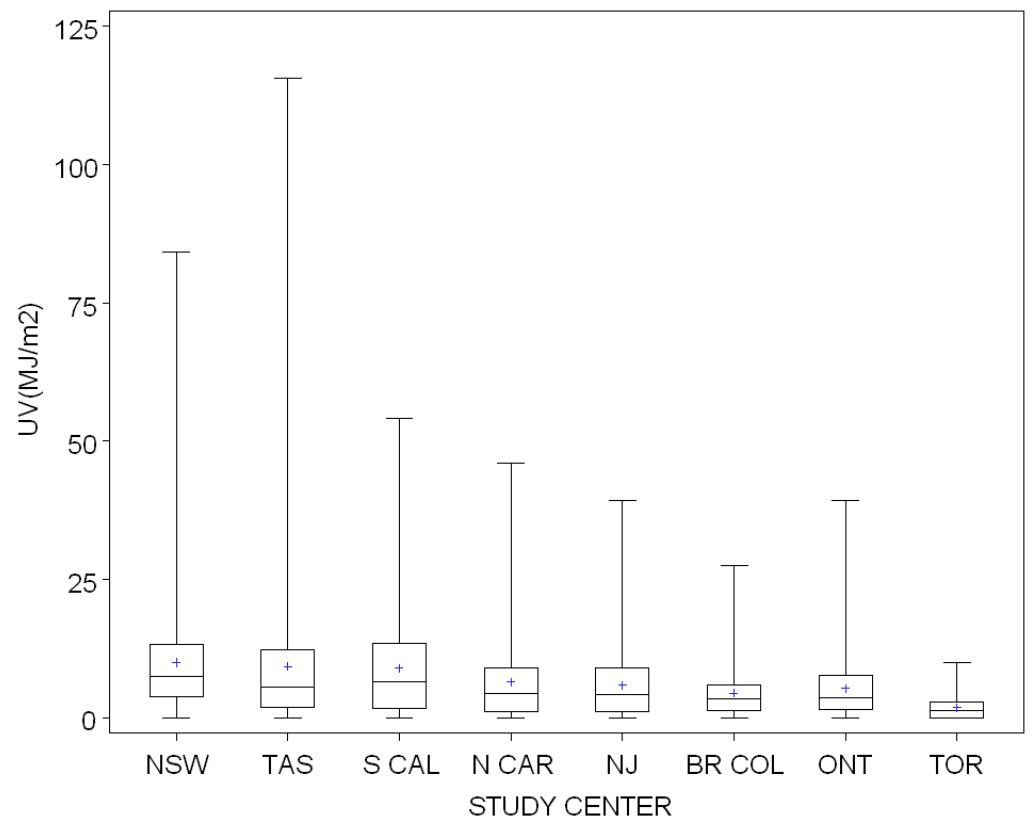

Figure 1.

Cumulative site-specific UVE dose in $\mathrm{MJ} / \mathrm{m}^{2}$ : Mean, median and interquartile range experienced at the body site by participants in GEM centers (NSW, New South Wales; TAS, Tasmania, Australia; S CAL, Southern California; N CAR, North Carolina; NJ, New Jersey, BR COL, British Columbia, Canada; ONT, Ontario, Canada; TOR, Torino, Italy). Site-specific UVE dose was calculated as total ambient UVE (estimated by NCAR for each participant's place of residence at each decade of age) for an assumed average 8 hours a day multiplied by the proportion of total sun exposure hours that the site was exposed for each participant. This sun exposure measure takes into account both site-specific sun exposure behavior and ambient UV irradiance. 


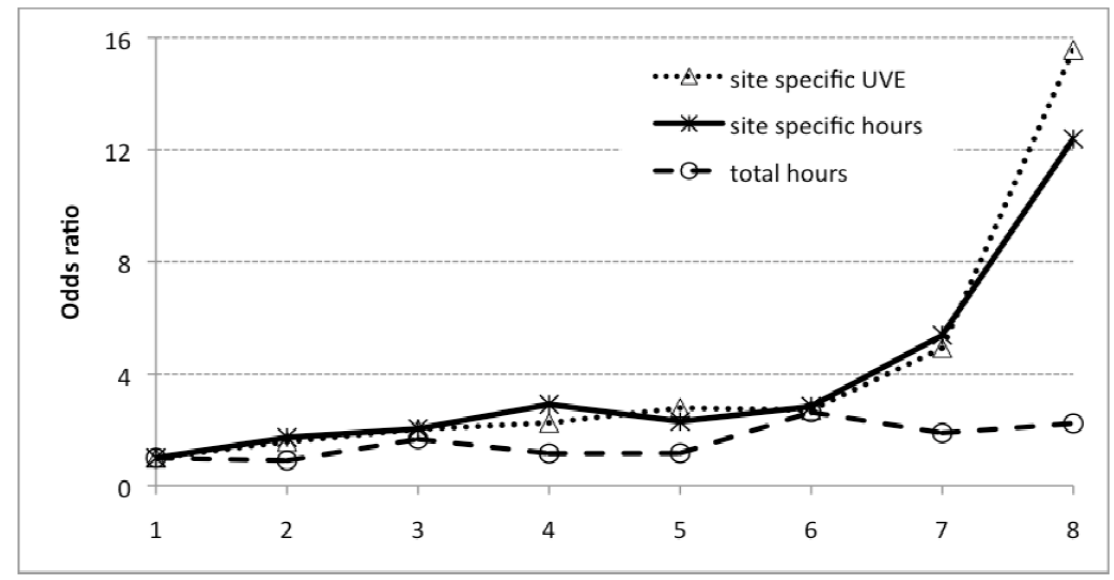

Figure 2.

ORs for solar elastosis on all body sites in 2,304 GEM participants: cumulative sun exposure measures in eight ordered categories containing equal numbers of participants in models adjusted for age, sex, center, pigmentary characteristics and nevi; site-specific exposure also adjusted for body site (head and neck, trunk, arms, legs). Total hours and site-specific hours of sun exposure were reported by participants; site-specific UVE was calculated as total ambient UVE for an assumed average 8 hours a day multiplied by the proportion of total sun exposure hours that the site was exposed for each participant. 


\section{Table 1}

Age, sex and bodysite in relationship to histologic solar elastosis in participants in the GEM study $(N=2,589)$

\begin{tabular}{|c|c|c|c|c|}
\hline \multirow[b]{2}{*}{ Characteristic } & \multicolumn{2}{|c|}{ Solar elastosis } & \multirow{2}{*}{$\begin{array}{l}\text { Present vs. absent } \\
\text { solar elastosis } \\
\text { OR }^{*}(95 \% \text { CI })\end{array}$} & \multirow[b]{2}{*}{$P$ value } \\
\hline & $\begin{array}{l}\text { Absent } \\
N=785\end{array}$ & $\begin{array}{l}\text { Present } \\
N=1,804\end{array}$ & & \\
\hline \multicolumn{5}{|l|}{ Sex } \\
\hline Female & 400 & 728 & 1.00 & \\
\hline Male & 385 & 1,076 & $0.99(0.81-1.21)$ & 0.93 \\
\hline \multicolumn{5}{|l|}{ Age } \\
\hline $11-40 y$ & 216 & 162 & 1.00 & \\
\hline $41-50 y$ & 185 & 210 & $1.78(1.30-2.43)$ & \\
\hline $51-60 y$ & 181 & 355 & $3.50(2.58-4.76)$ & \\
\hline $61-70 y$ & 102 & 434 & $8.18(5.84-11.45)$ & \\
\hline $71-97 y$ & 101 & 643 & $11.91(8.55-16.59)$ & $<0.001$ \\
\hline \multicolumn{5}{|l|}{ Age by sex } \\
\hline \multicolumn{5}{|l|}{ Male } \\
\hline $11-50 y$ & 141 & 151 & 1.00 & \\
\hline $51-70 y$ & 172 & 490 & $3.32(2.43-4.55)$ & \\
\hline $71-97 y$ & 72 & 435 & $7.16(4.96-10.34)$ & $<0.001$ \\
\hline \multicolumn{5}{|l|}{ Female } \\
\hline $11-50 y$ & 260 & 221 & 1.00 & \\
\hline $51-70 y$ & 111 & 299 & $4.10(2.97-5.65)$ & \\
\hline $71-97 y$ & 29 & 208 & $11.50(7.18-18.42)$ & $<0.001$ \\
\hline \multicolumn{5}{|l|}{ Body site } \\
\hline Trunk/Pelvis & 449 & 665 & 1.00 & \\
\hline Head/Neck & 30 & 438 & $9.26(6.08-14.11)$ & \\
\hline Arms & 71 & 409 & $5.32(3.84-7.35)$ & \\
\hline Legs & 229 & 283 & $0.99(0.76-1.29)$ & $<0.001$ \\
\hline
\end{tabular}

* OR for sex adjusted for age (continuous) and study center; ORs for age adjusted for sex and center; ORs for age by sex adjusted for center. ORs for body site adjusted for age (continuous), sex, and study center. 
Table 3

The relationships between solar elastosis and lifetime cumulative sun exposure measures in participants in the GEM study $(N=2,304)$.

\begin{tabular}{|c|c|c|c|c|}
\hline \multirow[b]{4}{*}{ Cumulative sun exposure } & \multicolumn{2}{|c|}{ Solar elastosis } & \multicolumn{2}{|c|}{ Present vs. absent solar elastosis } \\
\hline & \multirow{3}{*}{$\begin{array}{l}\text { Absent } \\
N=697\end{array}$} & \multirow{3}{*}{$\begin{array}{c}\text { Present } \\
N=1,607\end{array}$} & \multicolumn{2}{|c|}{ adjusted for pigmentary characteristics \& nevi } \\
\hline & & & no & yes \\
\hline & & & $\mathrm{OR}^{*}(95 \% \mathrm{CI})$ & $\mathrm{OR}^{\dagger}(95 \% \mathrm{CI})$ \\
\hline \multicolumn{5}{|l|}{ Site specific measurement ${ }^{\S}$} \\
\hline \multicolumn{5}{|c|}{ Site-specific sun exposure hours } \\
\hline Quartile 1 & 262 & 302 & 1.00 & 1.00 \\
\hline Quartile 2 & 215 & 365 & $1.66(1.24-2.21)$ & $1.80(1.34-2.41)$ \\
\hline Quartile 3 & 171 & 406 & $1.69(1.24-2.31)$ & $1.86(1.35-2.56)$ \\
\hline Quartile 4 & 49 & 534 & $4.33(2.87-6.53)$ & $5.12(3.35-7.83)$ \\
\hline$P$ for trend & & & $<0.001$ & $<0.001$ \\
\hline \multicolumn{5}{|c|}{ Site-specific beach and water UVE dose } \\
\hline None & 258 & 464 & 1.00 & 1.00 \\
\hline Tertile 1 & 208 & 328 & $1.24(0.93-1.66)$ & $1.32(0.99-1.78)$ \\
\hline Tertile 2 & 138 & 393 & $1.66(1.23-2.26)$ & $1.85(1.35-2.53)$ \\
\hline Tertile 3 & 104 & 443 & $2.06(1.50-2.83)$ & $2.37(1.70-3.30)$ \\
\hline$P$ for trend & & & $<0.001$ & $<0.001$ \\
\hline \multicolumn{5}{|l|}{ Site-specific UVE dose } \\
\hline Quartile 1 & 265 & 292 & 1.00 & 1.00 \\
\hline Quartile 2 & 232 & 348 & $1.53(1.15-2.04)$ & $1.66(1.24-2.23)$ \\
\hline Quartile 3 & 154 & 427 & $1.91(1.40-2.61)$ & $2.11(1.53-2.91)$ \\
\hline Quartile 4 & 46 & 540 & $4.41(2.91-6.69)$ & $5.20(3.40-7.96)$ \\
\hline$P$ for trend & & & $<0.001$ & $<0.001$ \\
\hline \multicolumn{5}{|c|}{ Non-site specific measurement } \\
\hline \multicolumn{5}{|l|}{ Total sun exposure hours } \\
\hline Quartile 1 & 276 & 284 & 1.00 & 1.00 \\
\hline Quartile 2 & 187 & 386 & $1.41(1.05-1.88)$ & $1.47(1.10-1.98)$ \\
\hline Quartile 3 & 143 & 456 & $1.66(1.20-2.29)$ & $1.75(1.26-2.43)$ \\
\hline Quartile 4 & 91 & 481 & $1.96(1.34-2.86)$ & $2.13(1.45-3.12)$ \\
\hline$P$ for trend & & & $<0.001$ & $<0.001$ \\
\hline \multicolumn{5}{|l|}{ Ambient UVE } \\
\hline Quartile 1 & 322 & 235 & 1.00 & 1.00 \\
\hline Quartile 2 & 186 & 365 & $1.05(0.72-1.54)$ & $1.07(0.73-1.58)$ \\
\hline Quartile 3 & 120 & 471 & $1.12(0.65-1.93)$ & $1.16(0.67-2.01)$ \\
\hline Quartile 4 & 69 & 536 & $1.23(0.59-2.55)$ & $1.29(0.61-2.70)$ \\
\hline$P$ for trend & & & 0.56 & 0.49 \\
\hline
\end{tabular}


$\S_{\text {Site-specific sun exposure measures also adjusted for body site of melanoma }}$ 